\title{
A Enfermagem de ReabilitaÇÃo e o Desmame Ventilatório numa Unidade de CuIDAdos INTENSIVOS
}

\author{
Rehabilitation Nursing And Ventilatory WeEaning in An InTEnsive Care Unit
}

\author{
ENFERMERÍA DE REHABILITACIÓN Y DESTETE VENTILATORIO EN UNIDAD DE CUIDADOS INTENSIVOS
}

DOI 10.33194/rper.2021.177; Data de Receção: 2021-06-16; Data de Aceitação: 2021-10-18

\author{
Rui Miguel Outeiro ${ }^{1} \mathbb{D}$; Salete Soares ${ }^{2} \mathbb{D}$ \\ ${ }^{1}$ ULSAM - Hospital de Santa Luzia, Viana Do Castelo, Portugal; ${ }^{2}$ IPVC Viana do Castelo, Portugal
}

Autor correspondente: Rui Miguel Outeiro, 2351@ulsam.min-saude.pt

\section{RESUMO}

Introdução: 0 sucesso desmame ventilatório é fundamental, traduzindo-se na redução do tempo de internamento nas Unidades de Cuidados Intensivos $(\mathrm{UCl})$, na minimização das sequelas da ventilação mecânica e na maximização da qualidade de vida, onde o Enfermeiro de Reabilitação (ER) assume um papel fundamental.

Metodologia: Estudo qualitativo, descritivo, exploratório. Participaram todos os ER (4)a exercer funções numa UCI da ARS Norte. Recorreu-se à entrevista semiestruturada e à observação não participada sistemática, após a qual se efetuou análise de conteúdo e cruzamento dos dados colhidos nas entrevistas e na observação. 0 objetivo é conhecer a perceção dos ER no desmame ventilatório.

Resultados: A reabilitação motora, respiratória e a avaliação da pessoa são intervenções identificadas pelos ER no cuidado à pessoa em desmame ventilatório, as quais devem ser iniciadas precocemente. As dificuldades identificadas prendem-se com a falta de material, ausência de um protocolo de desmame ventilatório, défice de interação entre a equipa e défice na continuidade de cuidados

Discussão: A intervenção do ER na pessoa em desmame ventilatório traduz-se em ganhos positivos tais como a minimização das sequelas da imobilidade, o aumento da força muscular, a capacitação para a autonomia e o célere desmame ventilatório. A elaboração de um instrumento de avaliação estruturado e sistematizado são elementos fulcrais neste processo

Conclusão: 0 desmame ventilatório é um processo muito específico onde a intervenção do ER requer a aquisição e desenvolvimento de competências muito próprias. Destaca-se a inclusão destes conteúdos na formação especializada, formação contínua e no reconhecimento de experiências em realidades semelhantes.

DESCRITORES: Respiração artificial, Desmame do respirador, Enfermagem, Reabilitação

\section{ABSTRACT}

Introduction: Successful ventilatory weaning is essential, translating into a reduction in the length of stay in the Intensive Care Units (ICU), in the minimization of the sequelae of mechanical ventilation and in the maximization of the quality of life, where the Rehabilitation Nurse (ER) plays a key role.

Methodology: Qualitative, descriptive, exploratory study. All ER (4) exercising functions in a UCI of ARS Norte participated. A semi-structured interview and systematic non-participated observation were used, after which a content analysis and crossing of data collected in the interviews and in the observation were carried out. The objective is to know the perception of RE in ventilatory weaning.

Results: Motor and respiratory rehabilitation and the assessment of the person are interventions identified by the RE in the care of the person undergoing ventilatory weaning, which should be started early. The identified difficulties are related to the lack of material, absence of a ventilatory weaning protocol, lack of interaction between the team and deficit in the continuity of care

Discussion: The intervention of the RE in the person undergoing ventilatory weaning translates into positive gains such as the minimization of immobility sequelae, increased muscle strength, training for autonomy and rapid ventilatory weaning. The development of a structured and systematized assessment instrument are key elements in this process 
Conclusion: Ventilatory weaning is a very specific process where the intervention of the RE requires the acquisition and development of very specific skills. The inclusion of these contents in specialized training, continuous training and in the recognition of experiences in similar realities is highlighted.

DESCRIPTORS: Respiration artificial, Respirator weaning, Nursing, Rehabilitation

\section{RESUMEN}

Introducción: El éxito del destete ventilatorio es fundamental, que se traduce en una reducción de la estancia en las Unidades de Cuidados Intensivos (UCl), en la minimización de las secuelas de la ventilación mecánica y en la maximización de la calidad de vida, donde la Enfermera de Rehabilitación (ER) juega un papel clave.

Metodología: Estudio cualitativo, descriptivo, exploratorio. Participaron todos los ER (4) que ejercían funciones en una UCI de la ARS del Norte. Se utilizó entrevista semiestructurada y observación sistemática no participada, luego de lo cual se realizó análisis de contenido y cruce de datos recolectados en las entrevistas y observación. El objetivo es conocer la percepción de la ER en el destete ventilatorio.

Resultados: La rehabilitación motora, respiratoria y la valoración de la persona son intervenciones identificadas por el ER en el cuidado de la persona sometida a destete ventilatorio, que deben iniciarse precozmente. Las dificultades identificadas están relacionadas con la falta de material, ausencia de protocolo de destete ventilatorio, falta de interacción entre el equipo y déficit en la continuidad asistencial

Discusión: La intervención del RE en la persona en destete ventilatorio se traduce en ganancias positivas como la minimización de secuelas de inmovilidad, aumento de la fuerza muscular, entrenamiento para la autonomía y destete ventilatorio rápido. El desarrollo de un instrumento de evaluación estructurado y sistematizado son elementos clave en este proceso.

Conclusión: El destete ventilatorio es un proceso muy específico donde la intervención del RE requiere la adquisición y desarrollo de habilidades muy específicas. Se destaca la inclusión de estos contenidos en la formación especializada, la formación continua y en el reconocimiento de experiencias en realidades similares.

DESCRIPTORES: Respiración artificial, Desconexión del Ventilador, Enfermería, Rehabilitación

\section{INTRODUÇÃO}

A Ventilação Mecânica Invasiva (VMI) é uma das intervenções de suporte vital em diversas situações clínicas, tais como a sépsis grave, falência respiratória, ARDS, no entanto não é inócua. Está associada a diversas complicações, razão pela qual, o processo de desmame ventilatório deve ser célere, seguro e com base na evidência científica ${ }^{(1)}$.

O desmame ventilatório é o processo de descontinuação do suporte ventilatório associado à remoção da via aérea artificial (2), constituindo esta fase como crucial, pois o seu insucesso obriga à reintubação, traduzindo-se num maior número de dias de internamento, de mortalidade e morbilidade hospitalar, com consequências económicas e sociais negativas para a população, pelo que deve ser realizado em condições seguras e o mais precoce possível.

O uso de protocolos de desmame ventilatório tem vindo a ser defendido por alguns autores ${ }^{(3,4,5)}$ no sentido de reduzir as variações na prática clínica e proporcionar uma prática baseada em evidência, existe evidência que aponta para o uso de protocolos como forma de reduzir o tempo de ventilação mecânica e de redução do tempo gasto no processo de desmame ventilatório ${ }^{(3)}$.

É de realçar, que a reabilitação suportada em protocolos, e voltada para a mobilização precoce em pessoas em situação crítica sob VMI por um período superior a $24 \mathrm{~h}$, é altamente recomendada (1). A execução de um plano de Reabilitação Respiratória (RR), na fase aguda da doença mantém e potencia as capacidades existentes no intuito de promover a independência funcional, a autonomia, e um desmame ventilatório mais célere, com um impacto direto na redução dos dias de internamento em Unidade de Cuidados Intensivos (UCI) ${ }^{(6)}$, pelo que, em virtude da sua eficácia, na maioria dos países desenvolvidos, a reabilitação está integrada nos cuidados gerais obrigatórios às pessoas internadas nestas unidades ${ }^{(4)}$.

A evidência diz-nos que um plano de RR que incorpore exercícios de reeducação funcional respiratória e reeducação funcional motora progressiva e precoce é benéfica para a pessoa internada em $\mathrm{UCI}$, com efeitos quer a nível funcional, psicológico e ventilatório, favorecendo o desmame ventilatório e a diminuição do tempo de ventilação mecânica ${ }^{(6)}$.

O Enfermeiro de Reabilitação (ER) participa e dá resposta aos problemas e limitações vivenciados pela pessoa em desmame ventilatório. A sua intervenção passa pela avaliação da pessoa, treino específico dos músculos respiratórios, reeducação diafragmática, treino de técnicas de limpeza das vias aéreas e de exercícios de reeducação funcional motora com vista a minimizar a fraqueza muscular e o descondicionamento cardiorrespiratório, promovendo uma melhoria da força muscular generalizada, do estado funcional e do sucesso do desmame ventilatório ${ }^{(7)}$. Para o alcance 
de tais objetivos é fulcral a existência de ER nas UCl e a sua participação efetiva em todo o processo de cuidados, nomeadamente, antes, durante e após o desmame ventilatório ${ }^{(8,9)}$

Portanto, consideramos pertinente conhecer a intervenção dos ER no desmame ventilatório, numa UCl da zona norte de Portugal, neste contexto, o estudo desenvolvido procura responder à seguinte questão: Qual a perceção dos ER de uma $\mathrm{UCl}$ sobre a sua intervenção no processo de desmame ventilatório?

\section{METODOLOGIA}

Para a concretização do objetivo proposto, optou-se por um estudo de natureza exploratória e descritiva com abordagem qualitativa, com recurso a uma amostra por conveniência. Os participantes do estudo são todos os enfermeiros especialistas em reabilitação (quatro) a exercer funções na UCl em estudo, sendo este o critério de inclusão previamente definido.

Como instrumentos de colheita de dados recorreu-se a entrevista semiestruturada e a observação não participada sistemática, cujos dados foram registados em grelha própria elaborada para o efeito.

O estudo decorreu numa $\mathrm{UCl}$ do tipo $\mathrm{B}$ situada num hospital da região norte de Portugal, no período de 1 de novembro a 30 de dezembro de 2018.

As entrevistas foram realizadas num gabinete respeitando a privacidade dos participantes. Foi realizada gravação áudio, após autorização dos entrevistados, e posteriormente transcritas.

Os dados recolhidos através da observação não participada, foram complementados com notas de campo com pormenores pertinentes para o estudo, registados em grelha própria.

Os conteúdos das entrevistas foram transcritos e submetidos à técnica de análise de conteúdo ${ }^{(10)}$, sendo por esta técnica que os discursos foram organizados em categorias e subcategorias.

Os dados recolhidos através da grelha de observação foram analisados de forma descritiva, tendo sido posteriormente efetuada a triangulação destes dados com os das entrevistas.

A colheita de dados decorreu durante dois meses, foram observados 3 dos 4 enfermeiros que prestam cuidados de enfermagem de reabilitação, atendendo a que um elemento estava ausente do serviço. Cada ER foi observado durante um turno (manhã) em 3 dias não consecutivos.

0 estudo cumpriu, em todo o seu processo, as recomendações da Declaração de Helsínquia. 0 estudo foi aprovado pela Comissão de Ética da Instituição onde se realizou o estudo com parecer n59/2018CES. Foi obtido o consentimento informado livre e esclarecido de todos os informantes.

\section{RESULTADOS}

Da análise das entrevistas emergiram 5 áreas temáticas e, em cada uma, três ou mais categorias (Quadro $n^{\circ} 1$ ).

A observação permitiu constatar que a reabilitação funcional respiratória e motora é uma prática sistemática no atendimento à pessoa em desmame ventilatório. No entanto, relativamente à avaliação da pessoa, esta já não acontece de forma sistematizada. Notou-se uma preocupação, de todos os participantes no estudo, em iniciar o mais precocemente a reeducação funcional motora, em consonância com critérios de segurança. Todas as pessoas em desmame ventilatório têm um plano de reeducação funcional motora implementado, embora não conste nesse plano a frequência e a intensidade dos exercícios, nem o registo do comportamento da pessoa em desmame ventilatório.

\section{DISCUSSÃO}

Relativamente à Intervenção do ER no processo de desmame ventilatório, os participantes realçaram a importância da reabilitação motora; reabilitação respiratória e avaliação da pessoa no sucesso do mesmo, também vários autores $(7,9)$ referem que os programas de reeducação funcional motora e respiratória nas UCl devem fazer parte dos cuidados prestados pelos ER com o objetivo de aumentar a força muscular e melhorar o estado funcional da pessoa, pois estão associados ao sucesso no desmame ventilatório. Importa salientar que embora não exista consenso quanto à intensidade, frequência e duração das intervenções, os programas estruturados e individualizados demonstram evidência na recuperação do doente crítico ${ }^{(11)}$ e salientaram a importância das técnicas de reeducação funcional respiratória na pessoa em desmame ventilatório, com o objetivo de melhorar a força dos músculos respiratórios, promover a eliminação de secreções e controlar a ansiedade ${ }^{(7,12)}$.

A avaliação da pessoa também é considerada pelos informantes como intervenção integrante no processo de desmame ventilatório. A avaliação da situação clínica deverá incluir a avaliação sintomatológica, com recurso ao exame físico, e meios complementares de diagnóstico, pertinentes para cada caso e contexto ${ }^{(12)}$. Deve ter-se em linha de conta a avaliação da capacidade funcional, função respiratória, função muscular, qualidade de vida, ansiedade e depressão (12). 


\begin{tabular}{|c|c|}
\hline ÁREA TEMÁTICA & CATEGORIAS \\
\hline \multirow{3}{*}{$\begin{array}{l}\text { Intervenção do enfermeiro de reabilitação no processo de desmame } \\
\text { ventilatório }\end{array}$} & Reabilitação motora \\
\hline & Reabilitação respiratória \\
\hline & Avaliação da pessoa \\
\hline \multirow{4}{*}{$\begin{array}{l}\text { Dificuldades do enfermeiro de reabilitação no processo de desmame } \\
\text { ventilatório }\end{array}$} & Ausência de protocolos \\
\hline & Défice de interação com a equipa \\
\hline & Défice na continuidade de cuidados \\
\hline & Falta de material \\
\hline \multirow{3}{*}{$\begin{array}{l}\text { Perceção dos enfermeiros de reabilitação sobre o sucesso do desmame } \\
\text { ventilatório }\end{array}$} & $\begin{array}{l}\text { Falta de experiência } \\
\text { Reducão do tempo de internamento }\end{array}$ \\
\hline & Minimização das sequelas \\
\hline & Implementação de protocolos \\
\hline \multirow{2}{*}{$\begin{array}{l}\text { Pertinência da intervenção enfermeiro de reabilitação no processo de } \\
\text { desmame ventilatório }\end{array}$} & Resultados positivos \\
\hline & Garantir a continuidade de cuidados \\
\hline \multirow{3}{*}{ Aquisição de conhecimentos específicos } & Curso de especialização \\
\hline & Formação contínua \\
\hline & Experiências em outras unidades \\
\hline
\end{tabular}

Os protocolos de atuação, no processo de desmame ventilatório, são um elemento fulcral, vários autores corroboram desta ideia ${ }^{(3,4)}$, pois consideram que estes contribuem para a padronização da atuação dos vários intervenientes, melhoram a condução do desmame, mantém um alto índice de sucesso e baixa mortalidade. Permitem, ainda definir as atividades e responsabilidades de cada interveniente com objetivos claros e bem precisos, contribuindo para a promoção da colaboração interprofissional.

Outra dificuldade mencionada foi a ausência de interação entre a equipa multidisciplinar por falta de comunicação e colaboração entre os elementos da equipa (fisiatra e fisioterapeuta), a pouca dinâmica de equipa e o desconhecimento dos restantes elementos da equipa do projeto dos ER. Sabemos que a tomada de decisão dinâmica e colaborativa tem influência em todo o processo de desmame ventilatório. Quando não há colaboração entre os profissionais envolvidos, o desmame pode ser fragmentado, demorado e inconsciente, resultando em atrasos e complicações ${ }^{(3)}$.

Outro constrangimento mencionado foi o défice na continuidade de cuidados de enfermagem de reabilitação. Os ER chamam a atenção, nas suas narrativas, para a necessidade de cuidados de enfermagem de reabilitação para além do período da manhã, pois o desmame ventilatório, a maioria das vezes, iniciam-se neste período, e prolongam-se para o período da tarde, acabando por a pessoa ser extubada no período em que não pode beneficiar de cuidados de enfermagem de reabilitação. No sentido de dar resposta às necessidades de cuidados de enfermagem de reabilitação e no respeito pelos padrões de qualidade, importa adequar e organizar a prestação dos cuidados para satisfazer as necessidades das pessoas ${ }^{(12)}$. Constatou-se que os participantes referem a falta de material, considerando-a imprescindível para a boa prática de cuidados, nomeadamente no treino dos músculos inspiratórios, pelo que consideram ser necessário a aquisição do treshold, peak flow meter e eletroestimulador

As pessoas submetidas à ventilação mecânica invasiva desenvolvem fraqueza muscular generalizada, essencialmente dos músculos inspiratórios, o que contribuiu para alterações respiratórias, nomeadamente a dispneia, que limita a recuperação funcional motora. 0 treino e a exercitação da musculatura inspiratória podem melhorar a força e a resistência muscular inspiratória durante e após o desmame ventilatório, melhorando potencialmente a dispneia e a qualidade de vida dessas pessoas ${ }^{(12)}$. Tanto o treshold como a electroestimulação são fulcrais neste processo.

o peak flow meter é usado como medidor de fluxo de ar expirado, é capaz de prever o sucesso da extubação e construir Scores que identifiquem a capacidade de uma tosse eficaz ${ }^{(13)}$. Assim, o uso deste equipamento ajuda na identificação das pessoas, com tosse eficaz, prevenindo a falha na extubação.

Sobre o sucesso do desmame ventilatório, constatou-se que os ER referem a pouca consciencialização dos enfermeiros sobre a redução do tempo de internamento associado ao sucesso de desmame ventilatório. A ideia de desmame ventilatório bem-sucedido associada à diminuição do tempo de internamento da pessoa nas UCI vai ao encontro do exposto por vários autores sobre a relação da falência da extubação ao maior tempo de hospitalização, maior frequência de traqueostomia e de complicações pulmonares, piores desfechos funcionais e maior mortalidade $(3,14)$.

Nas entrevistas é referida a importância da intervenção do ER na minimização das sequelas da ventilação mecânica invasiva. A intervenção do ER face à pessoa ventilada passa pela implementação precoce de um programa de reeducação funcional estruturado e multiprofissional com vista a otimização das funções cognitivas, respiratórias, 
neuromusculares e osteoarticulares favorecendo o desmame precoce e por sua vez minimizando o tempo de internamento e as suas sequelas. (7)

Os informantes referem a importância da elaboração e implementação de um protocolo, pois consideram que este contribui para um desmame bem-sucedido. Esta ideia é corroborada por dois estudos que referem que o desmame da ventilação realizado seguindo uma padronização traduz-se numa melhor condução, mantendo o alto índice de sucesso e baixa mortalidade ${ }^{(4,5)}$ e que a intervenção precoce na pessoa submetida a ventilação mecânica invasiva promove um desmame ventilatório célere e minimiza as sequelas do internamento na UCl. Este conceito é partilhado pelos autores ${ }^{(2)}$ que se referem à reabilitação precoce na $\mathrm{UCl}$, como forma de diminuir a dependência da ventilação mecânica, o tempo de desmame ventilatório e prevenir as alterações associadas à imobilidade.

Relativamente à pertinência da intervenção ER no processo de desmame ventilatório, os participantes consideram que esta intervenção produz resultados positivos e garante a continuidade de cuidados.

Os informantes são unanimes sobre a pertinência da sua intervenção, referindo-se aos resultados positivos obtidos com a sua intervenção. Esta ideia contrasta com a escassez de estudos nesta área, que demonstrem os ganhos em saúde da intervenção dos ER no processo de desmame ventilatório, na minimização das sequelas da ventilação mecânica invasiva e na redução do tempo de internamento nas UCI, embora seja salientada a importância de os cuidados de enfermagem de reabilitação serem diários e que garantam a continuidade dos cuidados de reabilitação.

Também, foi realçada a importância da aquisição de conhecimentos específicos e que estes emergem quer da formação ao longo do curso de especialização, mas também, da formação contínua e do conhecimento das experiências em outras unidades. Nas entrevistas constatamos que os enfermeiros de reabilitação fazem referência à aquisição de conhecimentos específicos para intervir junto da pessoa durante o desmame ventilatório, no decurso da sua formação especializada em reabilitação. O curso de especialização permite ao enfermeiro dotar-se de saberes e competências específicas no cuidar da pessoa em desmame ventilatório, capacitando-o para intervenções específicas da enfermagem de reabilitação, não apenas para manter as capacidades funcionais, mas para evitar mais incapacidades e prevenir complicações, bem como para defender o seu direito à qualidade de vida, em consonância com o preconizado nas competências especificas dos ER ${ }^{(15)}$.

A formação continua é referida como veículo para adquirir conhecimentos para fazer face às necessidades de intervenção junto da pessoa em desmame ventilatório e os informantes mencionaram ter procurado conhecimento através de livros especializados, congressos, entre outros. Percebeu-se ainda, que os enfermeiros procuram formação sobre electroestimulação, auscultação pulmonar, interpretação radiológica, drenagens posturais e sobre a utilização do cought-assist. Estes achados estão em consonância com o estudo sobre a Formação Profissional Contínua e Qualidade dos Cuidados de Enfermagem que faz referência à importância do ensino sequencial, baseado nos saberes como suporte para a aquisição de competências do cuidar, para um sistema em que a aquisição de competências, interagindo em permanência com os saberes adquiridos, se completaria no contexto de execução como ambiente formativo indispensável ${ }^{(16)}$. Os enfermeiros fazem referência à necessidade e dever de procurar outras experiências em contextos semelhantes para daí extrair modelos de intervenção a aplicar na sua realidade de intervenção.

\section{CONCLUSÃO}

A reeducação funcional respiratória e motora é referida nas entrevistas e comprovada na observação não participada como uma intervenção dos ER, como essencial para o êxito do processo de desmame ventilatório. No entanto, consideramos ser necessário o desenvolvimento de mais estudos no sentido de demonstrar evidência do impacto da reeducação funcional respiratória e motora praticada pelos ER, no processo de cuidados à pessoa em desmame ventilatório.

Os enfermeiros identificaram a avaliação da pessoa em desmame ventilatório como uma preocupação e intervenção dos ER, mas da observação constatou-se que esta não era uma prática sistematizada e global. Os resultados sugerem que esta deve ser integrada de forma sistematizada na prática diária e que a construção de um instrumento de recolha de dados adaptado para a pessoa submetida à VMI, onde conste a avaliação da capacidade funcional, função respiratória, função muscular, qualidade de vida, ansiedade e depressão, tendo ainda em conta a sintomatologia e os meios complementares de diagnóstico, poderá ser uma mais-valia.

As dificuldades referidas pelos ER durante o processo de cuidar da pessoa em desmame ventilatório, prendem-se essencialmente com a inexistência de um protocolo de desmame ventilatório, com o défice de interação com a restante equipa, com a falta da continuidade de cuidados, de material e, ainda, falta de experiência. Face aos obstáculos identificados, emerge a necessidade de elaboração de um protocolo de desmame ventilatório que envolva os ER, médicos, a restante equipa de enfermagem, nutricionista, fisiatra e fisioterapeuta.

Emerge deste estudo que a intervenção dos ER na pessoa em desmame ventilatório se traduz em ganhos positivos, nomeadamente, na minimização das sequelas da imobilidade; no aumento da força muscular; na capacitação para a autonomia e num célere e eficaz desmame ventilatório, mas, considera-se pertinente demonstrar evidência científica sobre ganhos em saúde resultantes da sua atuação, através de novos estudos em diferentes contextos de UCl. 
Este estudo apresenta, como principal limitação, o facto de os dados obtidos, não poderem ser generalizados, mas considera-se que a sua pertinência poderá constituir o estímulo para novos estudos sobre esta temática com um maior número de participantes, alargá-lo a outras UCI de forma a ser possível a generalização dos resultados e, por sua vez, demonstrar ganhos em saúde que emergem da prestação de cuidados especializados de enfermagem de reabilitação.

\section{REFERÊNCIAS BIBLIOGRÁFICAS}

1. Schmidt GA, Girard TD, Kress JP, Morris PE, Ouellette DR, Alhazzani W, et al. Liberation From Mechanical Ventilation in Critically Ill Adults. Chest [Internet]. Elsevier BV; 2017 Jan;151(1):160-5. Available from: http://dx.doi.org/10.1016/j.chest.2016.10.037

2. Vaz I M, Maia M, Melo, A MC et al. Desmame ventilatório difícil o papel da medicina física e de reabilitação. Med Port. 2011; 299-308. Available from: https://www.actamedicaportuguesa.com/revista/index.php/amp/article/viewFile/1618/1200

3. Savi A, Teixeira C, Maccari JG et al. Ventilação Mecânica. Fundamentos e Pratica Clínica: desmame da ventilação mecânica. $1^{\mathrm{a}}$ edição Rio de Janeiro: Brasil Roca;2016. 523-539p. ISNB 978-85-277-2808-9

4. Blackwood B, Burns K, Cardwell,C. R,O'Halloran P. Protocolized versus non-protocolized weaning for reducing the duration of mechanical ventilation in critically ill adult patients. Cochrane Database of Systematic Reviews. 2014 nov 6;1-81. Available from: http://dx.doi.org/10.1002/14651858.CD006904.pub3

5. Ambrosino N, Vitacca $M$. The patient needing prolonged mechanical ventilation: a narrative review. Multidisciplinary Respiratory Medicine.2018 Fev 26; 13(6). Available from https://doi.org/10.1186/s40248-018-0118-7

6. Santos A, Oliveira I, Silveira T. Mobilização precoce em UCl. Salutis Scientia, 2010; 2, 20-24 Available from: http: //www.salutisscientia.esscvp.eu/Site/Artigo.aspx?artigoid=30463

7. Cordeiro M, Menoita E. Manual de Boas Práticas na Reabilitação Respiratória: conceitos, princípios e técnicas. $1^{\mathrm{a}}$ ed. Loures: Lusociência ;2012.380p.

8. China Neves M F Avaliação do impacto da intervenção do Enfermeiro de Reabilitação com a utilização da Reeducação Funcional Respiratória no doente crítico, numa UCl. Coimbra (Portugal): Escola Superior de Enfermagem de Coimbra, CURSO DE MESTRADO EM ENFERMAGEM DE REABILITAÇÃO; Dez. 2015 Available from: http:// web.esenfc.pt/?url=lEuajd2b

9. Pereira J A Competências do enfermeiro de reabilitação com doentes dependentes no autocuidado em UCI. Porto (Portugal): Escola Superior de Enfermagem do Porto; 2012 Available from: http://hdl.handle.net/10400.26/9340

10. Hernández RS, Fernández CC, Baptista P I. Metodologia de pesquisa. $5^{\circ}$. Ed. São Paulo: McGrawHill;2013.629p

11. Azevedo P, Gomes B. Effects of early mobilisation in the functional rehabilitation of critically ill patients: a systematic review. Revista de Enfermagem Referência [Internet]. Health Sciences Research Unit: Nursing; 2015 Jun 30; №5(IV Série):129-38. Available from: http://dx.doi.org/10.12707/riv14035

12. Ordem dos Enfermeiros. Guia Orientador de Boas Práticas: reabilitacão respiratória. Lisboa: Ordem dos Enfermeiros; 2018 jan. Available from: https:/www.ordemenfermeiros.pt/media/5441/gobp_reabilita\%C3\%A7\%C3\%A3o-respirat\%C3\%B3ria_mceer_final-para-divulga\%C3\%A7\%C3\%A3osite.pdf

13. Gobert F, Yonis H, Tapponnier R, Fernandez R, Labaune M-A, Burle J-F, et al. Predicting Extubation Outcome by Cough Peak Flow Measured Using a Built-in Ventilator Flow Meter. Respiratory Care [Internet]. Daedalus Enterprises; 2017 Sep 12;62(12):1505-19. Available from: http://dx.doi.org/10.4187/respcare.05460

14. Reis H F C, Almeida MLO, Rocha M S. Extubation failure influences clinical and functional outcomes in patients with traumatic brain injury. J Bras Pneumol.2013 jun; 39(3):330-338. Available from: https://dx.doi.org/10.1590/S18037132013000300010

15. DR. Diário da República Eletrónico n. ${ }^{\circ} 85 / 2019$ Série II: Regulamento 392/2019. Lisboa 2019 mai. Available from: https: //dre.pt/web/guest/pesquisa/-/search/122216893/details/normal?l=1

16. Varandas M L, Lopes A. Formação profissional contínua e qualidade dos cuidados de enfermagem: a necessidade de uma mudança de paradigma educativo. Rev. Lusófona de Educação. 2012 maio 17; 22:141-158. Avaiable from: http://revistas.ulusofona.pt/index.php/rleducacao/article/view/3286

\section{DIVULGAÇÕES ÉTICAS}

Contribuição do(s) autor(es):

Conceptualização: RO, SS

Metodologia: RO, SS

Validação: RO, SS

Análise formal: RO, SS

Investigação: RO

Tratamento de dados: RO

Preparação do rascunho original: RO

Redação e edição: RO, SS

Revisão: RO, SS

Todos os autores leram e concordaram com a versão publicada do manuscrito.

Financiamento:

Este trabalho não recebeu nenhuma contribuição financeira ou bolsa.

Comissão de Ética:

Estudo autorizado pela Comissão de Ética PARA A SAÚDE PARECER №59/2018-CES).

Declaração de consentimento informado:

O consentimento informado por escrito para publicar este trabalho foi obtido dos participantes.

Conflitos de interesse:

Os autores não declaram nenhum conflito de interesses.

Proveniência e revisão por pares: 
Não comissionado; revisto externamente por pares.

(c) Autor (es) (ou seu (s) empregador (es)) e APER/RPER 2021. Reutilização permitida de

(c) $\$=$ acordo com CC BY-NC-ND. Nenhuma reutilização comercial. 